\title{
Design Method and Calibration of Moulinet*
}

\author{
Hirokazu ITOH**, Hirokazu YAMADA** and Sinsuke UDAGAWA** \\ ** Tokyo Metropolitan College of Industrial Technology \\ 8-17-1 Minami-senzyu, Arakawa-ku, Tokyo, Japan \\ E-mail: hito@acp.metro-cit.ac.jp
}

\begin{abstract}
The formula for obtaining the absorption horsepower of a Moulinet was rewritten, and the physical meaning of the constant in the formula was clarified. Based on this study, the design method of the Moulinet and the calibration method of the Moulinet that was performed after manufacture were verified experimentally. Consequently, the following was clarified; (1) If the propeller power coefficient was taken to be the proportionality constant, the absorption horsepower of the Moulinet was proportional to the cube of the revolution speed, and the fifth power of the Moulinet diameter. (2) If the Moulinet design was geometrically similar to the standard dimensions of the Aviation Technical Research Center's type-6 Moulinet, the proportionality constant $\boldsymbol{C}_{\boldsymbol{l}}$ given in the reference could be used, and the absorption horsepower of the Moulinet was proportional to the cube of the revolution speed, the cube of the Moulinet diameter, and the side projection area of the Moulinet. (3) The proportionality constant $\boldsymbol{C}_{\boldsymbol{I}}$ was proportional to the propeller power coefficient $\boldsymbol{C}_{\boldsymbol{P}}$.
\end{abstract}

Key words: Technology Transfer and Education, Measurement, Aeronautical Engineering, Similarity Law, Internal Combustion Engine, Moulinet, Propeller, Power, Measurement, Calibration

\section{Introduction}

A Lycoming O-320-E2D introduced in 1983 for the educational experiment was found to have exceeded the operation standards (e.g., the cylinder temperature of the No. 4 cylinder exceeded the standard) and still showed good durability. The engine was sent to the FAA certified repair shop in California in March, 2009, and a full overhaul was carried out.

As a result of the visual inspection of the Moulinet used in the engine, some cracks were discovered near the root portion of the Moulinet wing. In order to judge whether it could be repaired or not, the depth of the cracks was measured by X-ray inspection, and it was judged that repair was possible. Then, after the circumference of the cracks was excised and laminating adhesion using the same material (mahogany) was carried out, the form was restored by CNC processing.

When the design data and the experimental results of the Moulinet were scrutinized and the method of designing the Moulinet was reinvestigated, it turned out that a lack of explanation and misprints were found in Yoshii ${ }^{(1)}{ }^{(3)}$, and the unknown matter had increased.

Furthermore, obtaining information about the design is impossible due to the advanced age of the engineer also in the company that had requested the manufacturing of the 
Moulinet, and this technology may become unavailable due to this situation.

In this paper, the absorption horsepower formula of the Moulinet was rewritten, the physical meaning of the constant in the formula was clarified, and the formula was arranged to optimize its practical use in the design method of the Moulinet. Then, the calibration method of the Moulinet performed after manufacture was verified experimentally.

\section{Measurement principle of propeller absorption horsepower by Moulinet}

Brake power when a piston engine is equipped with a propeller is called propeller absorption horsepower $\boldsymbol{P}$. If the same propeller is used, since the propeller diameter $\boldsymbol{D}$ and the propeller power coefficient $\boldsymbol{C}_{\boldsymbol{P}}$ (defined below) can be considered to be fixed, the propeller absorption horsepower is proportional to the cube of the propeller revolution speed $\boldsymbol{N}$. If air density, which changes with atmospheric conditions, is corrected for, simple measurement of the maximum brake power of the test engine can be carried out by measuring the propeller revolution speed.

The propeller exclusively designed for measurement of the maximum brake power of the test engine is called the Moulinet. On stand-testing of the standard engine which a brake power is known to, the absorption horsepower curve of Moulinet should be measured beforehand. If this preparation is made, the brake power when a test engine is equipped with the Moulinet can be determined by counting backward from the propeller revolution speed.

\section{Rewriting of Moulinet absorption horsepower formula}

If the torque $\boldsymbol{Q}$ required to rotate a Moulinet is assumed to be a function of the revolutions $\boldsymbol{n}$ per unit time, the representative length $\xi$, and the air density $\rho$, it will be expressed with empirical formula (1), which sets the non-dimensional proportionality constant of $\boldsymbol{C}$.

$$
Q=C_{q} \rho^{x} n^{y} \xi^{z}
$$

From $\boldsymbol{x}, \boldsymbol{y}$ and $\boldsymbol{z}$ having equal dimensions in both neighborhoods,

$$
\begin{aligned}
& \text { Left side }=\left(\mathrm{kg} \cdot \frac{\mathrm{m}}{\mathrm{sec}^{2}} \cdot \mathrm{m}\right)=(\mathrm{kg})(\mathrm{sec})^{-2}(\mathrm{~m})^{2} \\
& \text { Right side }=\left(\frac{\mathrm{kg}}{\mathrm{m}^{3}}\right)^{x}\left(\frac{1}{\mathrm{sec}}\right)^{y}(\mathrm{~m})^{z}=(\mathrm{kg})^{x}(\mathrm{sec})^{-y}(\mathrm{~m})^{z-3 x}
\end{aligned}
$$

Hence,

$$
\left.\begin{array}{rlc}
\mathrm{kg} & : & 1=x \\
\mathrm{sec} & : & -2=-y \\
\mathrm{~m} & : & 2=z-3 x
\end{array}\right\}
$$

These simultaneous equations are solved as $\boldsymbol{x}=1, \boldsymbol{y}=2, \boldsymbol{z}=5$; therefore the torque $\boldsymbol{Q}$ can be expressed with formula (3).

$$
Q=C_{q} \rho n^{2} \xi^{5}
$$

If $\Omega[\mathrm{rad} / \mathrm{s}]$ is the angular velocity of the Moulinet, the absorption horsepower of the Moulinet $\boldsymbol{P}[\mathrm{W}]$ can be expressed as follows.

$$
P=Q \Omega
$$

Here, 


$$
\begin{aligned}
& \Omega=2 \pi n \\
& P=C_{q} \rho n^{2} \xi^{5} \cdot 2 \pi n=2 \pi C_{q} \cdot \rho n^{3} \xi^{5}
\end{aligned}
$$

According to the reference (6) edited by the Japan Aeronautical Engineers' Association, the Moulinet diameter $\boldsymbol{D}$ is taken to represent the length $\xi$, and $2 \pi \boldsymbol{C} \boldsymbol{q}$ represents the propeller power coefficient $\boldsymbol{C}_{\boldsymbol{P}}$. When the Moulinet absorption horsepower is expressed using the propeller power coefficient, it will be proportional to the cube of the revolution speed and the fifth power of the Moulinet diameter as shown in the following formula.

$$
\begin{aligned}
& C_{P}=2 \pi C_{q} \\
& \therefore \quad P=C_{P} \cdot \rho n^{3} D^{5}
\end{aligned}
$$

The dimension of $\xi^{5}$ in formula (4) is the fifth power of the length. Therefore, if the Moulinet diameter is set to $\boldsymbol{D}$ and the representative area of the Moulinet is set to $\boldsymbol{A}, \xi^{5}$ will be transposed to $\boldsymbol{A} \boldsymbol{D}^{3} \cdot \boldsymbol{C}^{\prime}$. Furthermore, when the number of revolutions per unit time $\boldsymbol{n}[1 / \mathrm{sec}]$ is transposed to $N / 1000[\mathrm{rpm} / 1000]$,

$$
\begin{aligned}
& P=2 \pi C_{q} \cdot \rho\left(\frac{N}{1000}\right)^{3} \frac{1000^{3}}{60^{3}} A D^{3} \cdot C^{\prime}=C_{1} \cdot \rho A D^{3}\left(\frac{N}{1000}\right)^{3} \\
& C_{1}=2 \pi C_{q} \frac{1000^{3}}{60^{3}} C^{\prime}=C_{P} \frac{1000^{3}}{60^{3}} C^{\prime}
\end{aligned}
$$

Formula (6) becomes a formula from the Mechanical Engineering Handbook and Ogura $^{(4)-(5)}$, and the Moulinet absorption horsepower will be proportional to the cube of the revolution speed and the cube of the Moulinet diameter. The proportionality constant $\boldsymbol{C}_{\boldsymbol{l}}$ is proportional to the product of the propeller power coefficient $\boldsymbol{C}_{\boldsymbol{P}}$ and $\boldsymbol{C}^{\prime}$, and $\boldsymbol{C}^{\prime}$ is the constant for distributing $\xi^{5}$ to $\boldsymbol{A}$ and $\boldsymbol{D}^{3}$.

It was difficult in the days of Yoshii ${ }^{(1)-(3)}$ to analytically calculate the value of $\boldsymbol{C}_{\boldsymbol{I}}$ affected by the form of a Moulinet. Therefore, Yoshii manufactured the Moulinet in a standard form and obtained $\boldsymbol{C}_{\boldsymbol{I}}$ experimentally. Although these values can be used since the values of $\boldsymbol{C}_{\boldsymbol{I}}$ were published in the original text of Yoshii ${ }^{(1)-(3)}$ and in the Mechanical Engineering Handbook and Ogura ${ }^{(4)-(5)}$ for six kinds of standard forms, no description of how to obtain $\boldsymbol{A} . \quad \boldsymbol{A}$ is only described as "the total area of a blade."

Since the value of $\boldsymbol{C}_{\boldsymbol{l}}$ is based on the absorption horsepower $\boldsymbol{P}$ of the Moulinet and must have been obtained by counting backward using formula (6), the value of $\boldsymbol{C}_{\boldsymbol{l}}$ also changes based on how $\boldsymbol{A}$ is obtained. Although the wings area (in this case, the projection area seen from the axis of rotation front) was taken to be the representative area in propeller theory, when the rotation torque of the Moulinet was thought to have originated in the force of the object placed into the flow, the projection area seen from the side may have been used.

$\boldsymbol{C}_{\boldsymbol{I}}$ was determined by counting backward using $\boldsymbol{A}$ in formula (6) into the front projection area or the side projection area, and the results compared with the values given in the reference are shown in Table 1. Therefore, the value of $\boldsymbol{C}_{\boldsymbol{1}}$ given in the reference was concluded to be what was used to compute $\boldsymbol{A}$ as the side projection area. Since this consideration of how to obtain $\boldsymbol{A}$ and the design of the Moulinet mentioned in the following chapter were done simultaneously, the calculation used in Table 1 was performed using the type-6 Moulinet (refer to Fig. 1).

In order to achieve power measurement accuracy using the Moulinet, the official approved experiment which used the standard engine with a known brake power must be performed. However, if a Moulinet with a bigger absorption horsepower than that required can be designed, it can be adjusted to the required absorption horsepower by reducing the 
diameter, and one trial production can be managed.

Table 1 Representative Area $\boldsymbol{A}$ and Proportionality Constant $\boldsymbol{C}_{\boldsymbol{1}}$ (Refer to Fig.1 for Dimension of the Moulinet. )

\begin{tabular}{|c|c|c|}
\hline \multicolumn{3}{|c|}{ Condition of Calculation } \\
\hline$P$ & $\mathrm{HP}$ & 150 \\
\hline$N$ & $\mathrm{rpm}$ & 2700 \\
\hline$D$ & $\mathrm{~m}$ & 1.225 \\
\hline$M$ & $\mathrm{~m}$ & 0.155 \\
\hline$B$ & $\mathrm{~m}$ & 0.106 \\
\hline$\rho$ & $\mathrm{kg} / \mathrm{m}^{3}$ & 1.226 \\
\hline \multicolumn{3}{|c|}{ Proportionality Constant $\boldsymbol{C}_{\boldsymbol{1}}$ when $\boldsymbol{A}$ is } \\
\hline \multicolumn{2}{|c|}{$A=D \cdot M$} & 17.8 \\
\hline \multicolumn{2}{|c|}{$A=D \cdot B$} & 26.0 \\
\hline \multicolumn{2}{|c|}{$\begin{array}{l}\text { Given in the } \\
\text { reference }\end{array}$} \\
\hline
\end{tabular}
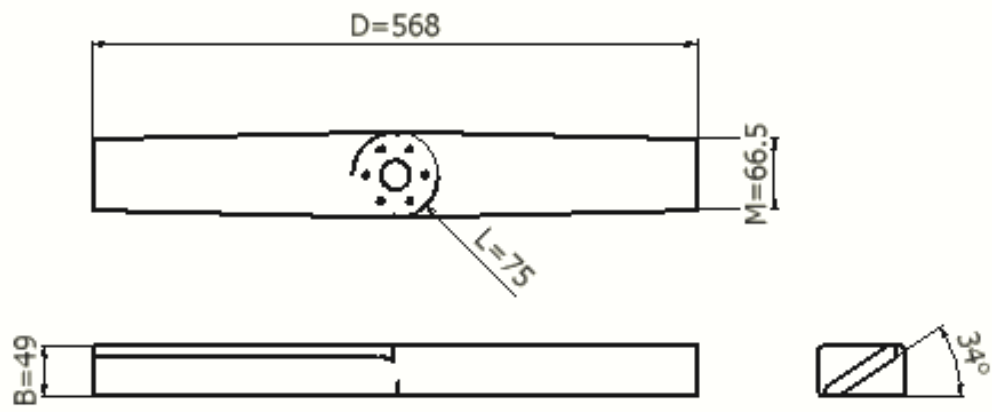

Fig.1 Standard Dimension of Type-6 Moulinet (Unit is mm)

\section{Designing procedure of type-6 Moulinet}

The geometric similarity conditions in the design deduced from the standard size of the type-6 Moulinet shown in Fig. 1 serve as formula (8). The type-6 Moulinet of Yoshii ${ }^{(1)}$ - (3) was described as an type-E Moulinet in the Mechanical Engineering Handbook and Ogura ${ }^{(4)-(5)}$.

$$
\begin{aligned}
& \frac{D}{B}=\frac{0.568}{0.049}=11.592 \\
& \frac{L}{M}=\frac{0.075}{0.0665}=1.128 \\
& \frac{M}{B}=\frac{0.0665}{0.049}=1.357 \\
& \alpha=34[\mathrm{deg}]
\end{aligned}
$$

Here, $\alpha$ is the pitch angle of the wing, $\boldsymbol{D}$ is the Moulinet diameter, $\boldsymbol{B}$ is the depth of the wing, $\boldsymbol{M}$ is the width of the wing, and $\boldsymbol{L}$ is the diameter of the boss part. If the value of 
$\boldsymbol{C}_{1}$ given in the reference is used, the unit of the absorption horsepower $\boldsymbol{P}$ of the Moulinet computed by formula (6) will be [HP]. Since $\boldsymbol{C}_{\boldsymbol{I}}$ is 26.2 in the type- 6 case, the value of $\boldsymbol{C}_{\boldsymbol{I}}$ is set to $26.2 \times 0.7457=19.537$ in order to convert it into [kW].

The air density $\rho$ at atmospheric pressure $101.3[\mathrm{kPa}]$ and temperature $25.0[\mathrm{degC}]$ is $1.226\left[\mathrm{~kg} / \mathrm{m}^{3}\right], \boldsymbol{A}$ is the side projection area $\left[\mathrm{m}^{2}\right]$ of the Moulinet, and $\boldsymbol{N}$ is the propeller revolution speed $[\mathrm{rpm}]$. Since the rated output and the revolution speed on the sea surface of the Lycoming O-320-E2D which is not equipped with the reduction gear are $112[\mathrm{~kW}]$ and 2700 [rpm], respectively, the design conditions of the Moulinet are an absorption horsepower of $112[\mathrm{~kW}]$ at $2700[\mathrm{rpm}]$.

To obtain the Moulinet diameter $\boldsymbol{D}$ from formula (6),

$$
D^{3}=\frac{P}{C_{1} \cdot \rho A} \cdot \frac{1}{\left(\frac{N}{1000}\right)^{3}}
$$

Hence,

$$
A=D \cdot B=D \frac{D}{11.592}=\frac{D^{2}}{11.592}
$$

Therefore,

$$
\begin{aligned}
& D^{3}=\frac{P}{C_{1} \cdot \rho \frac{D^{2}}{11.592}} \cdot \frac{1}{\left(\frac{N}{1000}\right)^{3}} \\
& \therefore D^{5}=\frac{11.592 \cdot P}{C_{1} \cdot \rho} \cdot \frac{1}{\left(\frac{N}{1000}\right)^{3}}=\frac{11.592 \times 112}{19.537 \times 1.226} \cdot \frac{1}{\left(\frac{2700}{1000}\right)^{3}}=2.7538 \\
& \therefore D=\sqrt[5]{2.7538}=1.225[\mathrm{~m}]
\end{aligned}
$$

The depth $\boldsymbol{B}$ was set to $\boldsymbol{D} / 11.592=0.106[\mathrm{~m}]$ based on the similarity conditions of formula (8). Since the diameter $\boldsymbol{L}$ of the boss part was not related to the calculation of the absorption horsepower of the Moulinet, it was determined from the pitch circle diameter of the propeller attachment bolt and the mechanical strength of the boss part. Since the pitch circle diameter of the propeller attachment bolt of the test engine was $120[\mathrm{~mm}]$ and the diameter of the bolt was $7 / 16$ [inch], the thickness from the perimeter of the bolt hole to the boss outside circumference was $22[\mathrm{~mm}]$, and the diameter $\boldsymbol{L}$ of the boss part was set to 0.175 [m]. The width $\boldsymbol{M}$ of the Moulinet was set to $\boldsymbol{L} / 1.128=0.155$ [mm] based on the similarity conditions of formula (8).

The design value and the actual value of the actually manufactured Moulinet are shown in Table 2. When the propeller absorption horsepower was counted backward from the design value of Table 2, it was set to $112[\mathrm{~kW}]$ as shown in formula (9).

$$
\begin{aligned}
& P=C_{1} \cdot \rho \cdot A \cdot D^{3}\left(\frac{N}{1000}\right)^{3}=C_{1} \cdot \rho \cdot B \cdot D \cdot D^{3}\left(\frac{N}{1000}\right)^{3} \\
& =19.537 \times 1.226 \times 0.106 \times 1.225^{4}\left(\frac{2700}{1000}\right)^{3}=112[\mathrm{~kW}]
\end{aligned}
$$

The manufactured Moulinet incorporated the laminating adhesion of two or more sheets of mahogany using epoxy resin in the direction of the axis, and processed with the CNC Fraise Machine. The diameter $\boldsymbol{D}$ was manufactured to be about $15 \%$ greater than the required size, as shown in Table 2. The diameter that corresponded to the rated revolution 
speed of $2700[\mathrm{rpm}]$ was determined experimentally using the test engine equipped with the Moulinet operated at full throttle, while the diameter was decreased unit by unit until the desired revolution speed was obtained.

Table 2 Designed and manufactured Dimensions of Moulinet

\begin{tabular}{|c|c|c|c|c|c|c|}
\hline \multirow{2}{*}{ Symbol } & \multicolumn{2}{|c|}{ Designed } & \multicolumn{2}{|c|}{ Manufactured } & \multicolumn{2}{|c|}{ Adjusted } \\
\hline & $\mathrm{m}$ & $\mathrm{kW}$ & $\mathrm{m}$ & $\mathrm{kW}$ & $\mathrm{m}$ & $\mathrm{kW}$ \\
\hline$D$ & 1.225 & \multirow{4}{*}{112} & 1.424 & \multirow{4}{*}{201} & 1.248 & \multirow{4}{*}{119} \\
\hline$B$ & 0.106 & & 0.104 & & 0.104 & \\
\hline$L$ & 0.175 & & 0.175 & & 0.175 & \\
\hline$M$ & 0.155 & & 0.143 & & 0.143 & \\
\hline
\end{tabular}

\section{Adjustment of Moulinet absorption horsepower}

The absorption horsepower estimated from the survey size at the completion of the manufacture of the Moulinet is 201 [kW]. Reducing the diameter unit by unit, the result which performed full throttle operation is shown in Fig. 2. It was confirmed experimentally that the engine revolution speed increased to 2015 [rpm] at the diameter $1.424[\mathrm{~m}]$, and increased to $2423[\mathrm{rpm}]$ at the diameter $1.306[\mathrm{~m}]$. The engine revolution speed was finally set to $2698[\mathrm{rpm}]$ at the diameter $1.248[\mathrm{~m}]$.

Although the Moulinet absorption horsepower at the final diameter was determined by counting backward to be $119[\mathrm{~kW}]$, since it was thought that the new test engine had generated a rated output of $112[\mathrm{~kW}]$ at $2700[\mathrm{rpm}]$, the proportionality constant $C_{I}$ of the actually manufactured Moulinet was corrected as follows.

$$
C_{1}^{\prime}=\frac{112}{119} C_{1}=\frac{112}{119} \times 19.537=18.388
$$

Since the air density compensation was made at the time of the experiment, it is thought that the correction was needed because the Moulinet was not manufactured under exactly the same conditions, since the details of the form of the type- 6 Moulinet are unknown.

In order to verify the validity of formula (9), the revolution speed and the diameter (data from Fig. 2) which were obtained in the experiment were substituted into formula (9), and the absorption horsepower of the Moulinet for every diameter was calculated and compared with the full throttle performance curve of the tested Lycoming engine. The result is shown in Fig. 3. The absorption horsepower values of the Moulinet before the proportionality constant $\boldsymbol{C}_{\boldsymbol{I}}$ produced under conditions that were not identical to the similarity form of the type-6 Moulinet ( $\triangle$ symbols), and after correction ( $\bigcirc$ symbols) were computed and are illustrated in Fig. 3. Moreover, an error range of $\pm 5 \%$ is displayed on the $\bigcirc$ symbols in the figure. Fig.3 shows that the absorption horsepower of the Moulinet is smaller than the net horsepower (the Lycoming operated at full throttle) in all revolution speed ranges. However, it turns out that the error of the absorption horsepower of the Moulinet is within $\pm 5 \%$ from 2600 [rpm] to 2700 [rpm].

On the other hand, before correction ( $\triangle$ symbols), the estimated absorption horsepower of the Moulinet was larger than the net horsepower at revolution speeds of 2500 [rpm] or more, and this contradicts its having operated at 2698 [rpm]. The formula for calculating the absorption horsepower $\boldsymbol{P}$ of the Moulinet which finished above adjustment is shown in formula (11). 


$$
\begin{aligned}
& P=C_{1} \cdot \rho \cdot B \cdot D \cdot D^{3}\left(\frac{N}{1000}\right)^{3} \\
& =18.388 \times 1.226 \times 0.104 \times 1.248^{4}\left(\frac{N}{1000}\right)^{3}=5.6874\left(\frac{N}{1000}\right)^{3}[\mathrm{~kW}]
\end{aligned}
$$

Three Moulinets whose designs were geometrically similar to the type-6 Moulinet were manufactured. The diameter was reduced, the full throttle operating examination was performed, and the diameter which corresponded to the rated revolution speed of the test engine was deduced. Here, if it is assumed that the brake power is proportional to the revolution speed (torque is constant) when full throttle operation of the aircraft engine is carried out, the relation $\boldsymbol{D}^{4} \boldsymbol{N}^{2}=$ Constant can be derived from formula (9).

Then, the common logarithms of the revolution speed were placed along the horizontal axis, the common logarithms of the diameter were placed along the vertical axis, and the result of the full throttle operating examination that involved reducing the diameter was plotted on Fig. 4. The $\diamond$ symbols in the figure show the results of the experiment conducted using the Moulinet designed for the Lycoming O-290-D2(rated output is 101 $[\mathrm{kW}]$ at $2600[\mathrm{rpm}])$, the $\bigcirc$ symbols represent the results of the experiment conducted using the Moulinet designed for the Lycoming O-320-E2D(rated output is 112 [kW] at 2700 $[\mathrm{rpm}]$ ), in which the design method described in the preceding chapter was introduced, and the $\triangle$ symbols represent the results of the experiment conducted using the Moulinet designed for the Continental C-145(rated output is 108 [kW] at 2700 [rpm]). Although each test engine is a horizontal opposite engine, the Lycoming are 4-cylinder engines and the Continental is a 6-cylinder engine.

Here, the inclinations of the straight lines determined by the $\bigcirc$ and $\triangle$ symbols are -0.503 and -0.504 , respectively. From this, it could be said that the revolution speed $N$ of the test engine and the Moulinet diameter $\boldsymbol{D}$ were related as follows: $\boldsymbol{D}^{4} \boldsymbol{N}^{2}=$ Constant. The Moulinet whose results are shown by $\triangle$ symbols showed a speed of 2680 [rpm], which was closed to the rated revolution speed for the diameter of 1241 [mm].

On the other hand, the inclination of the line determined by the $\diamond$ symbols is -0.383 and could not be regarded as the theoretical value $(-0.500)$. This engine caused the seizure of the crank metal during the experiment, and the diameter adjustment was not completed. The maximum revolution speed when the $112[\mathrm{~kW}](2700[\mathrm{rpm}])$ overhauled new engine was equipped with the same Moulinet as that represented by the $\bigcirc$ symbols was sufficient at 2680 [rpm] when full throttle operation was carried out. This result showed reproducibility with an error of \pm 50 [rpm]. 


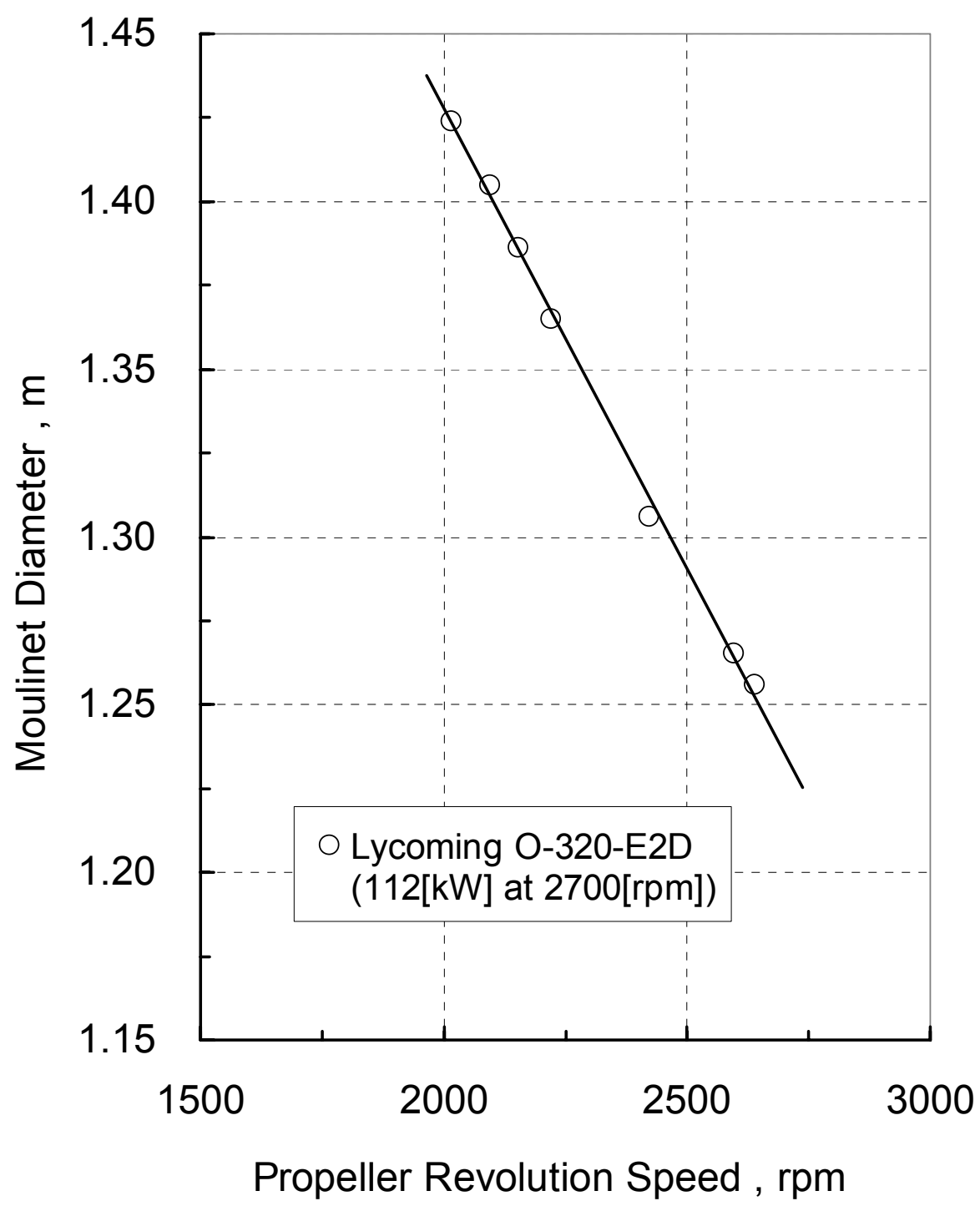

Fig.2 Propeller Revolution Speed obtained by Moulinet Diameter-shortened Operation (Lycoming O-320-E2D produces $112[\mathrm{~kW}]$ at 2700[rpm]) 


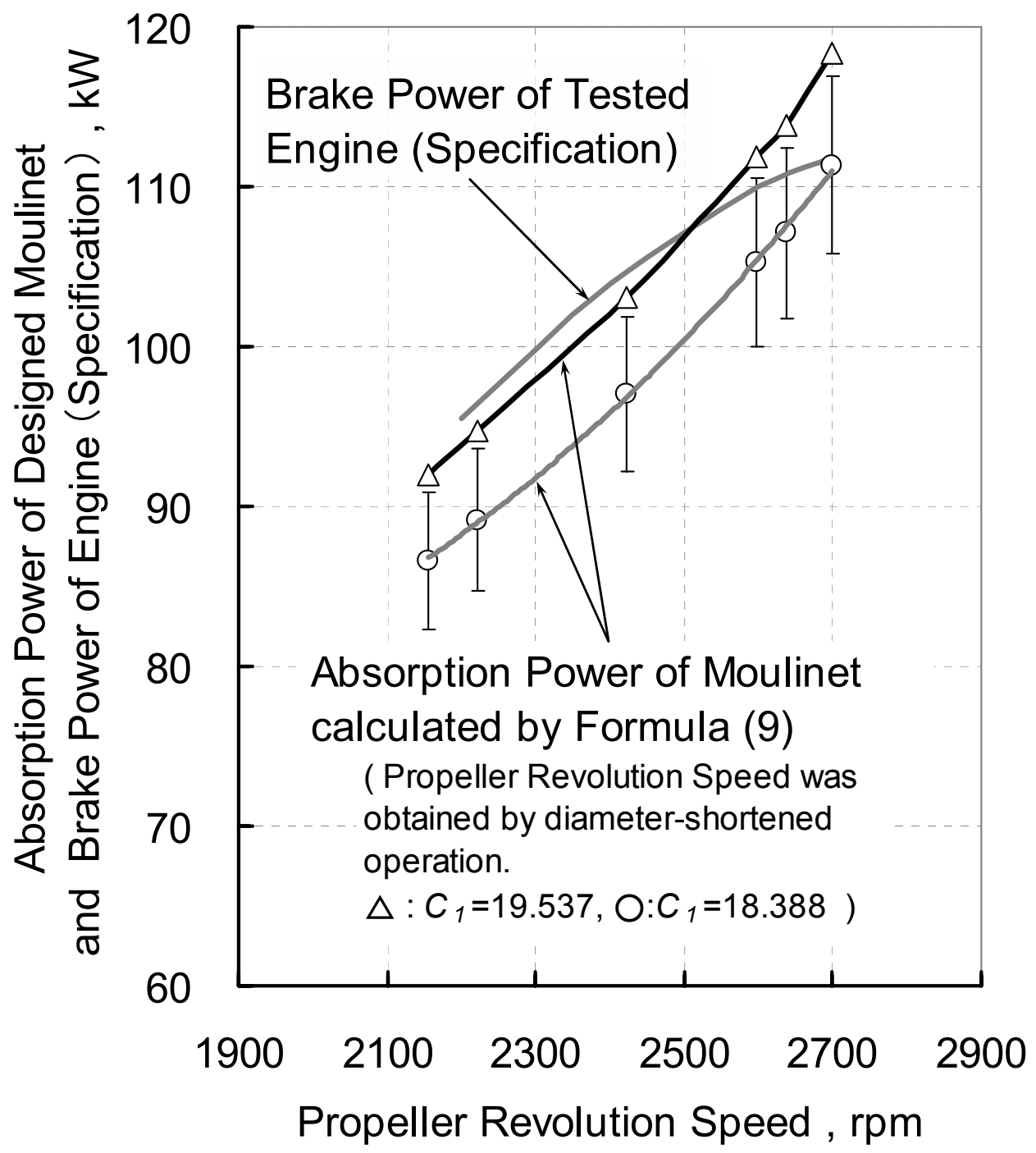

Fig.3 Propeller Revolution Speed versus Absorption Power of Designed Moulinet calculated by Formula (9) and Brake Power of Tested Engine (Specification of Lycoming O-320-E2D) 


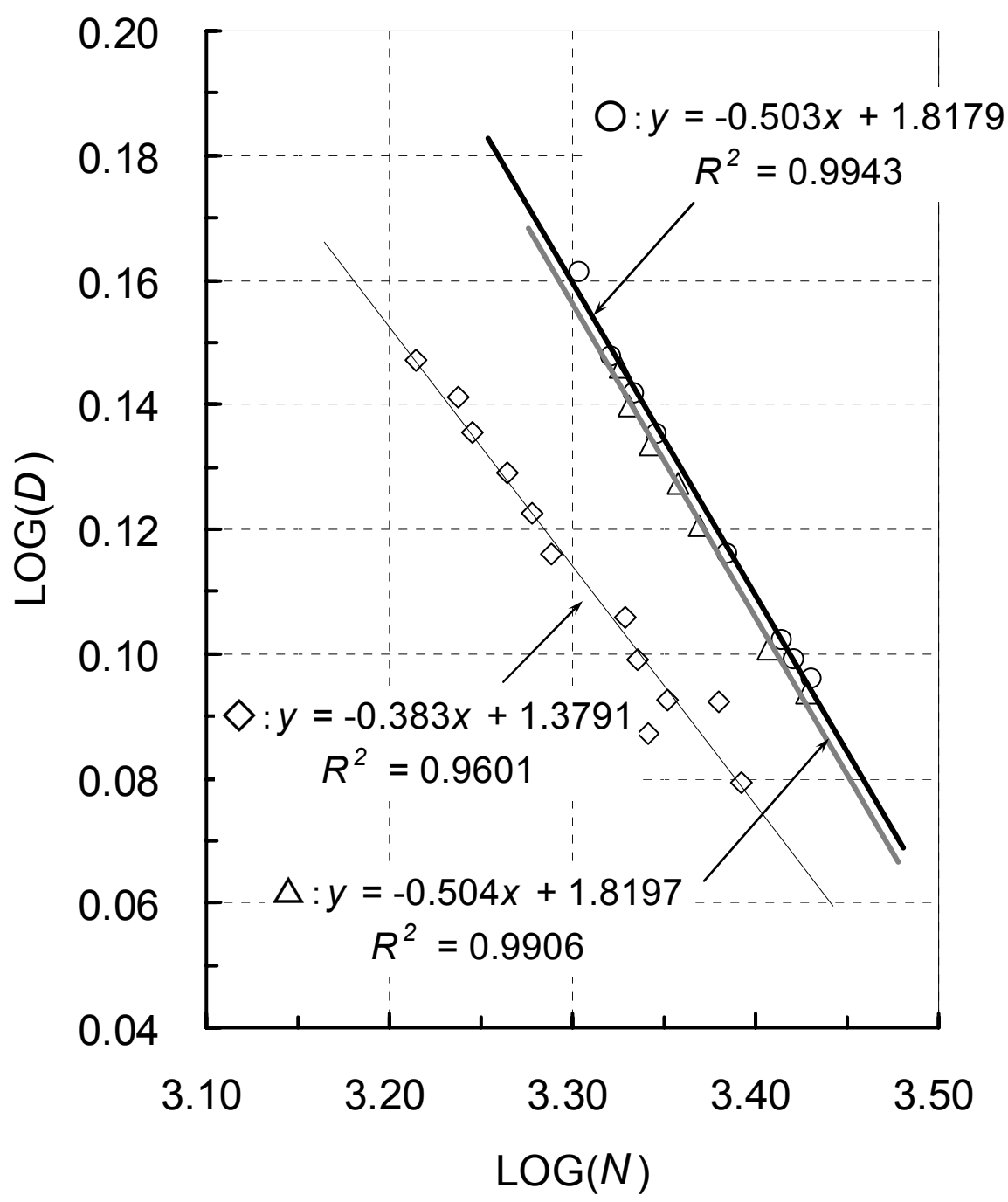

Fig.4 Common Logarithm of Propeller Revolution Speed versus Common Logarithm of Moulinet Diameter 


\section{Conclusion}

The formula for obtaining the absorption horsepower of the Moulinet was rewritten, and the physical meaning of the constant in the formula was examined. Consequently:

(1) If the propeller power coefficient was taken to be the proportionality constant, the absorption horsepower of the Moulinet was proportional to the cube of the revolution speed and the fifth power of the Moulinet diameter;

(2) If the Moulinet had a geometrically similar design with the Aviation Technical Research Center's standard-size type-6 Moulinet, the proportionality constant $\boldsymbol{C}_{\boldsymbol{I}}$ given in the reference could be used, and the absorption horsepower of the Moulinet was proportional to the cube of the revolution speed, the cube of the Moulinet diameter, and the side projection area of the Moulinet;

(3) The proportionality constant $\boldsymbol{C}_{\boldsymbol{I}}$ was proportional to the propeller power coefficient $C_{P}$.

Based on this study, the design method of the Moulinet and the calibration method performed after the manufacture of the Moulinet were verified experimentally. Since three Moulinets whose designs were geometrically similar to the Aviation Technical Research Center's type-6 Moulinet were actually manufactured, and the absorption horsepower of the Moulinet was experimentally verified with more than two engines with known rated output.

\section{Acknowledgments}

We are grateful to Mr. Katuo Ogura and Mr. Yoshie Ebata (former Professor of Tokyo Metropolitan College of Aeronautical Engineering) who provided suitable advice. We also express gratitude to Mr. Takashi Suzuki (former Vice-president of HINO MOTORS, LTD.) who offered precious references.

\section{References}

(1) Masao Yoshii, etc., "Experiment on Air Dynamometer", Miscellaneous Records of the Aviation Technical Research Center, Vol.14, (1925), pp.359-369

(2) Masao Yoshii., "Experiment on Air Dynamometer", Miscellaneous Records of the Aviation Technical Research Center, Vol.15, (1925), pp.384-393

(3) Masao Yoshii., "Experiment on Air Dynamometer", Miscellaneous Records of the Aviation Technical Research Center, Vol.22, (1926), pp.219-221

(4) Edited by The Japan Society of Mechanical Engineers., Mechanical Engineering Handbook, Vol.3, (1951), p.52-53, The Japan Society of Mechanical Engineers Press.

(5) Katuo Ogura., Revision Aeronautical Engine (1983), p.116-117, Kyoritu Press.

(6) Edited by Japan Aeronautical Engineers'Association., New Aeronautical Engineering Course Propeller (1988), p.23-32, Japan Aeronautical Engineers'Association Press. 